rest 3 mothers did not use any supplementation nor any other drugs. One baby had also anencephaly and was lost on the 2nd day of life. The rest babies had hydrocephalus, corpus callosum agenesis, meningomyelocele and underwent surgery. One baby has serious feeding problems thus is on nasogastric feeding. The surviving babies are between 10-26 months, all having physical rehabilitation. Physically the 4 babies are below 3rd percentile both for weight and height. In conclusion, occipital encephalocele is a life-threatening cranial anomaly. The overall outcome of the patient depends on the site and dimension of the lesion, as well as presence of accompanying congenital anomalies. Close multidisciplinary follow-up is needed. FA supplemantation should be nationally provoked.

\section{SEVEN DAY ANHEPATIC SURVIVAL IN A 19 MONTH OLD CHILD, AN INTERDISCIPLINARY CHALLENGE}

doi:10.1136/archdischild-2012-302724.0578

${ }^{1} \mathrm{H}$ Ringe, 'V Varnholt, ${ }^{2} \mathrm{~W}$ Luck, ${ }^{3} \mathrm{G}$ Puhl. ${ }^{1}$ Paediatric Intensive Care; ${ }^{2}$ Paediatric Gastroentrology; ${ }^{3}$ Department of General, Visceral, and Transplantation Surgery, Charite Campus Virchow, Berlin, Germany

Objective Description of pediatric intensive care and surgical management in a 19 month old child after primary liver-graftnon-function, who was managed anhepatic for 8 days in total and re-transplanted twice.

Case Report A 19 month old boy, $10 \mathrm{~kg}$ bodyweight, with ALF of unknown origin received an adult left liver lobe. After all vessels were connected and re-opened the graft showed a massive swelling and perfusion failure due to fulminant micro-vascular rejection and was removed immediately. The portal vein was attached end-toside to the cava inferior. Thereafter diffuse intra-abdominal bleeding occurred, requiring PPSB, factor VII, mass-transfusion and tranexamic-acid and the child was admitted to PICU sedated and ventilated.

To maintain ammonium, bile acids, bilirubin, and cerebral perfusion within thresholds continuous-single-pass-albumin-dialysis (SPAD) on turnover rates up to $150 \mathrm{ml} / \mathrm{kg} / \mathrm{h}$ of hemodiafiltration/filtration was used in total to bridge the anhepatic boy to his first (7 days) and second re-transplantation (1 day). Fresh-frozen-plasma to avoid hemorrhage, water-soluble vitamins, and amino-acids were continuously replaced.

Overall 16 surgical interventions (increased intra-abdominal pressure, portal vein kinking, portal and arterial thrombosis (second graft), removal of mesenterial lymphoid cysts, bile-duct-leak, second re-transplantation with cavo-portal anastomosis, and secondary abdominal wall closure with dermal-porcine-collagen, skinmesh-grafts) and anticoagulation with argatroban were needed to save the boy.

During the 6 month total hospital stay, including 6 weeks on mechanical ventilation, multiple bacterial, viral and fungal infections were detected that required early and timely antimicrobiological treatment.

At 1.5 year follow up the child was alive with intact graft and showed no neurologic sequelae.

\section{CENTRAL VENOUS CATHETER COLONISATION AND CATHETER RELATED SEPSIS: LESSONS LEARNT FROM EXIT SITE SKIN SWAB}

doi:10.1136/archdischild-2012-302724.0579

IV Ponnusamy, ${ }^{2} \mathrm{~V}$ Venkatesh, ${ }^{2} \mathrm{~A}$ Curley, ${ }^{3} \mathrm{~A}$ Perperoglou, ${ }^{4} \mathrm{~N}$ Brown, ${ }^{5} \mathrm{C}$ Tremlett, 'P Clarke. 'Neonatal Intensive Care Unit, Norfolk and Norwich University Hospitals NHS Foundation Trust, Norwich; ${ }^{2}$ Neonatal Intensive Care Unit, Cambridge University Hospitals NHS Foundation Trust, Cambridge; ${ }^{3}$ Norwich Medical School, University of East Anglia, Norwich; ${ }^{4}$ Clinical Microbiology, Cambridge University Hospitals NHS Foundation Trust, Cambridge; ${ }^{5}$ Department of Microbiology, Norfolk and Norwich University Hospitals NHS Foundation Trust, Norwich, UK
Background and Aim Percutaneous central venous catheters (PCVCs) are commonly inserted in neonates after topical antisepsis Presence of a PCVC is a risk factor for catheter-related sepsis (CRS). We examined the relationship between bacteriology of exit site skin swabs (ESSS) taken at line removal and line colonisation/CRS.

Design/methods For all PCVCs removed, ESSS and three separate PCVC segments (proximal, middle and tip) were sent for bacteriological culture. For clinically-septic neonates a peripheral blood culture was additionally obtained. PCVC colonisation was defined as a positive growth in any PCVC segment from a well neonate. Definite CRS was defined as positive growths with the same organism in any PCVC segment plus the blood culture from a clinically-septic neonate.

Results ESSS were culture-positive for 39/187 (21\%) lines removed. Univariate analysis showed that with a positive ESSS, line colonisation was 8 times higher (log odds ratio 2.13 [95\%CI: 1.18-3.08], $\mathrm{p}<0.001$ ), and definite CRS was 14 times higher (2.63 [1.14-4.14], $\mathrm{p}<0.005)$. Adjusting for various covariates, multivariate analysis using a logistic regression model confirmed an increased risk of CRS with a positive ESSS (log odds ratio 2.00 [95\%CI: 0.44-3.58], p=0.01). Conclusion Positive ESSS correlate strongly with PCVCcolonisation and definite CRS. Improved topical antisepsis, skin and catheter care is required to reduce the risk of colonised skin insertion sites associated with catheter placement, and the consequent risks of line colonisation and subsequent CRS.

\section{A SYSTEMATIC APPROACH TO PREVENTING CENTRAL VENOUS CATHETER-ASSOCIATED BLOODSTREAM INFECTION IN PATIENTS RECEIVING HOME PARENTERAL NUTRITION}

doi:10.1136/archdischild-2012-302724.0580

$1 \mathrm{~J}$ Gray, ${ }^{2} \mathrm{C}$ Holden, ${ }^{2} \mathrm{E}$ Sexton, ${ }^{3} \mathrm{~S}$ Pearce. ${ }^{1}$ Department of Microbiologyl; ${ }^{2}$ Nutritional Care; Infection Control, Birmingham Children's Hospital NHS Foundation Trust, Birmingham, UK

Background and Aims Central venous catheter-associated bloodstream infection (CBSI) is a serious complication in home parenteral nutrition (HPN) patients. Prevention of CBSI in hospitals is well-established, but in the home environment presents additional challenges. We applied hazard analysis and critical control points principles to develop a systematic approach to preventing HPNrelated CBSI. Here we describe the corrective actions and their clinical impact.

Methods Factors predisposing to infection, and corrective actions were identified through consensus by a multidisciplinary group of healthcare professionals between April and June 2012. The impact of these actions on CBSI was observed.

Results Key corrective actions were:

1. Staphylococcus aureus nasal screening and decolonisation where appropriate.

2. Multidisciplinary discharge planning meetings included consideration of patients' microbiology histories and optimisation of treatment of comorbidities (e.g. atopic eczema).

3. Increased monitoring of line access and care practices at home, aiming to attain the same standards as in hospital.

4. Launch of updated illustrated guide to identification and management of line site problems.

5. Streamlining of microbiology result reporting to facilitate early treatment of local infections.

6. Taurolidine locks for one patient with a poor line infection history.

Between April 2011 and March 2012 there were 13 CBSI acquired outside hospital, and during planning and implementation of corrective actions (April-June 2012) there were 4 CBSI. By contrast there was only one CBSI during the in the nine months following full implementation. 